\title{
STAT1 expression and HPV16 viral load predict cervical lesion progression
}

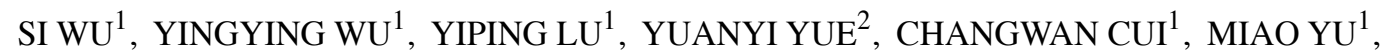 \\ SHUANG WANG ${ }^{1}$, MIAO LIU $^{1}$, YING ZHAO $^{3}$ and ZHENGRONG SUN ${ }^{1}$ \\ Departments of ${ }^{1}$ Biobank and ${ }^{2}$ Gastroenterology, Shengjing Hospital of China Medical University, Shenyang, \\ Liaoning 110004; ${ }^{3}$ Medical Examination Center, Shenyang Red Cross Hospital, Shenyang, Liaoning 110013, P.R. China
}

Received January 15, 2020; Accepted June 26, 2020

DOI: $10.3892 / \mathrm{ol} .2020 .11889$

\begin{abstract}
Cervical cancer is the fourth leading cause of cancer-associated mortality worldwide. However, its underlying molecular mechanisms are unclear. It is important to explore these mechanisms in order to identify novel diagnostic and prognostic biomarkers. The present study determined the association between STAT1 and human papillomavirus (HPV)16 in cervical lesions. STAT1 expression was detected by immunohistochemistry. Quantitative PCR was used to detect HPV16 viral load and STAT1 expression in cervical lesions. The potential associations among STAT1 expression, HPV16 viral load and the severity of cervical lesions in patients were analyzed using receiver operating characteristic (ROC) curves. The Cancer Genome Atlas database was used to analyze STAT1 expression and survival. High STAT1 expression was observed in 10.71 (3/28), 41.18 (14/34), $53.06(26 / 49)$ and $90.00 \%(27 / 30)$ of normal tissue, low-grade squamous intraepithelial lesion (LSIL), high-grade squamous intraepithelial lesion (HSIL) and cervical squamous cell carcinoma samples, respectively. The HPV16 copy number gradually increased with the progression of cervical lesions, with the highest copy number observed in cervical cancer samples. In addition, STAT1 expression was positively correlated with HPV16 viral load. Furthermore, ROC curve analysis demonstrated that the combination of STAT1 expression and HPV16 viral load was able to differentiate between LSIL/HSIL and cervical cancer samples. Bioinformatics analysis revealed that STAT1 expression was associated with improved survival in cervical cancer. Additionally, STAT1 expression was positively associated with the progression of cervical lesions, and HPV16 viral load may affect STAT1 expression. Overall,
\end{abstract}

Correspondence to: Professor Zhengrong Sun, Department of Biobank, Shengjing Hospital of China Medical University, 36 Sanhao, Heping, Shenyang, Liaoning 110004, P.R. China

E-mail: sunzr@sj-hospital.org

Key words: STAT1, cervical lesion/cervical cancer, human papillomavirus strain 16 , viral load, bioinformatics these findings indicate that STAT1 may be an indicator of the status of cervical lesions.

\section{Introduction}

Cervical cancer is the fourth leading cause of cancer-associated mortality worldwide, puts an increasing burden on low- and high-income countries alike, and was associated with $>500,000$ new cases and 300,000 deaths worldwide in 2018. Organized screening and human papillomavirus (HPV) vaccination can effectively reduce the incidence and mortality of cervical cancer (1). However, the mortality rate shows an increasing trend in underdeveloped and developing countries (2). Cervical cancer is commonly caused by high-risk human papillomavirus (HR-HPV) infection, and HPV DNA is found in $>90 \%$ of malignant cervical lesions. HPV16 and 18, as HR-HPV, account for $70 \%$ of cases, and HPV16 is the most common in female patients with cervical cancer (3). Therefore, it is essential to study the mechanism of HPV16 infection-induced cervical cancer.

Squamous cell carcinoma is the most common type of cervical cancer, accounting for $\sim 75 \%$ of cases, and involves a series of precancerous lesion stages, namely, low-grade squamous intraepithelial lesion (LSIL) and high-risk squamous intraepithelial lesion (HSIL) (4). Persistent infection with high-risk HPV can lead to malignant transformation of the normal cervix, although this can take several years to decades (5-7). HPV infection can lead to a tissue inflammatory response, activating the Janus kinase (JAK)/STAT signaling pathway and promoting HR-HPV replication (8). Previous studies have mainly focused on the association between HPV16 and STAT3, whereas few studies have considered STAT1 (9-12).

JAK/STAT signaling is involved in numerous physiological cell processes, such as proliferation, differentiation, apoptosis and immune system regulation; however, aberrant STAT regulation may lead to pathological events, including malignant cell transformation and metastasis (13). STAT1 was the first STAT protein identified in the interferon (IFN) signal transduction pathways, and is a 91-kDa cytosolic protein that adopts a structure with six highly conserved domains (14). It serves important roles in cytokine-induced signaling pathways, and can act as an antiviral and antibacterial mediator, growth inhibitor and 
inducer of apoptosis (13). STAT1 may have different roles in the progression of cancer. For example, various studies have indicated that STAT1, as a tumor suppressor, restrains the proliferation of cancer cells, including lung cancer cells, esophageal squamous cell carcinoma cells and colorectal cancer cells (15-17). In addition, STAT1 knockout mice are more susceptible to experimentally induced tumors, including the development of mammary adenocarcinoma (18). By contrast, aberrant STAT1 expression has been identified in human cancer, including pleural mesothelioma, renal cell carcinoma and breast cancer (19-21). Patients with high levels of STAT1 and/or pSTAT1 in cancer tissues have poor clinical outcomes compared with patients with low levels $(22,23)$. However, to the best of our knowledge, the effect of STAT1 on the progression of cervical cancer remains unknown.

The present study used human cervical tissues to investigate the associations among STAT1 expression, human cervical lesion progression and HPV16 viral load. The present study aimed to determine the association between STAT1 expression and the grade of cervical disease in HPV-infected patients.

\section{Materials and methods}

Study population. The present study included 141 cervical tissue specimens from patients treated at Shengjing Hospital, China Medical University (Shenyang, China) between January 2012 and December 2017. The specimens were divided into four groups: Normal, LSIL, HSIL and cancer. In the normal, LSIL and HSIL groups, cold knife conization was used to obtain the samples, while in the cancer group, samples were obtained during hysterectomy. The age of patients ranged between 20 and 70 years (median age, 43 years). Each HPV16-infected cervical tissue specimen underwent HPV flow-through hybridization detection and paraffin histopathological evaluation. The histopathological diagnosis was performed by two pathologists, the percentage of abnormal cells was recorded and the formalin-fixed, paraffin-embedded (FFPE) cervical specimens were graded histologically. All samples were collected with written informed consent from patients, and all related procedures were approved by the Ethics Committee of Shengjing Hospital, China Medical University (approval no. 2019PS476K).

HPV genotyping. An HPV GenoArray test kit (Hybribio Ltd.) was used to extract genomic DNA and perform HPV genotyping according to the manufacturer's protocols. The quality of the extracted DNA was ascertained by an internal control ( $\beta$-globin primers), which was included in PCR mix. The amplification protocol was as follows: $9 \mathrm{~min}$ of denaturation at $95^{\circ} \mathrm{C}$ and 40 cycles of $20 \mathrm{sec}$ of denaturation at $95^{\circ} \mathrm{C}$, $30 \mathrm{sec}$ of annealing at $55^{\circ} \mathrm{C}$, and $30 \mathrm{sec}$ of elongation at $72^{\circ} \mathrm{C}$, followed by a final extension for $5 \mathrm{~min}$ at $72^{\circ} \mathrm{C}$. This method was based on a flow-through hybridization principle and is able to classify $21 \mathrm{HPV}$ genotypes, including 13 high-risk subtypes $(16,18,31,33,35,39,45,51,52,56,58,59$ and 68), 5 low-risk subtypes $(6,11,42,43$ and 44$)$ and 3 subtypes that are common in China (53, 66 and CP8304) (24).

Immunohistochemical staining. STAT1 expression was examined using FFPE specimens. The tissue slices $(3-\mu \mathrm{m}$ thick) were deparaffinized in xylene and hydrated in an alcohol gradient series (100\% for $10 \mathrm{~min}, 95 \%$ for $5 \mathrm{~min}, 85 \%$ for $5 \mathrm{~min}$ and $75 \%$ for $5 \mathrm{~min})$. Specimens were boiled in citrate buffer (10 mM; pH 6.0) at $120^{\circ} \mathrm{C}$ for $5 \mathrm{~min}$. Subsequently, $3 \%$ hydrogen peroxide was added dropwise and sections were incubated for $20 \mathrm{~min}$ at room temperature. After blocking in 5\% BSA for $30 \mathrm{~min}$ at room temperature, STAT1 primary antibody (cat. no. AHO0832; dilution, 1:200; Thermo Fisher Scientific, Inc.) was used, and PBS was used as a negative control in place of the primary antibody. Subsequently, the sections were incubated in a refrigerator at $4^{\circ} \mathrm{C}$ overnight. The sections were washed with PBS and were incubated with horseradish peroxidase secondary antibody (cat. no. PV-6000; ZSGB-BIO,Inc.) at room temperature for $30 \mathrm{~min}$. Subsequently, 3,3'diaminobenzidine was added for $30 \mathrm{sec}$ for staining, and hematoxylin was used to counterstain the nuclei for $20 \mathrm{sec}$ at room temperature. Finally, neutral gum mounting medium was used to seal the slides and images were captured with a DP70 digital camera-equipped light microscope (Olympus Corporation). The score for each tissue image was evaluated by two independent pathologists and the average score was used as the final score. A total of 10 high-power fields were randomly observed for each specimen, and the percentage of positively stained cells in each high-power field was calculated and scored. The interpretation criteria were the product of the staining intensity and the staining area score. The staining intensity was scored on a scale of 1-4: 1, no staining; 2 , weak staining; 3, medium staining; 4 , strong staining. The percentage of stained cells was scored on a scale of $1-4: 1,<5 \% ; 2,5-25 \%$; $3,25-50 \% ; 4, \geq 50 \%$ ). The final product score was determined by multiplying these two values to obtain a score between 1 and 16: $<6$, low expression (-); $\geq 6$, high expression (+).

Reverse transcription-quantitative $(R T-q) P C R$. Frozen tissue samples from 20 patients per group were selected for RT-qPCR. Total RNA was extracted from human tissues using the GeneJET RNA Purification kit (cat. no. K0731; Thermo Fisher Scientific, Inc.) according to the manufacturer's protocol. The cDNA was synthesized using a Prime Script RT-PCR kit (Takara Bio, Inc.). Samples were incubated with reverse transcriptase at $37^{\circ} \mathrm{C}$ for $15 \mathrm{~min}$ and $85^{\circ} \mathrm{C}$ for $5 \mathrm{sec}$, and the reverse program stopped at $4^{\circ} \mathrm{C}$. qPCR assays were performed using a SYBR Premix ExTaq kit (Takara Bio, Inc.) and a 7,500-sequence detector (Applied Biosystems, Inc.). Amplification conditions were as follows: Initial step at $95^{\circ} \mathrm{C}$ for $30 \mathrm{sec}$, followed by 40 cycles of $95^{\circ} \mathrm{C}$ for $5 \mathrm{sec}$ and $60^{\circ} \mathrm{C}$ for $34 \mathrm{sec}$. The sequences of the primers were as follows: STAT1, forward 5'-CTTACCCAGAATGCCCTG AT-3', reverse 5'-CGAACTTGCTGCAGACTCTC-3'; and GAPDH, forward 5'-ACTGCCAACGTGTCAGTGGT-3' and reverse 5'-GTGTCGCTGTTGAAGTCAGA-3'. GAPDH was used as the normalizing gene for determination of $\triangle \mathrm{CT}$ values. Fold changes in gene expression were compared using $2^{-\Delta \Delta \mathrm{CT}}$ values (25).

DNA isolation and HPV16 viral load quantification. DNA isolations in the FFPE samples were performed using a Qiagen FFPE kit (56404; Qiagen $\mathrm{GmbH}$ ) as previously described (26). The present study evaluated the expression levels of HPV16 L1 to represent HPV16. Using GAPDH as an 

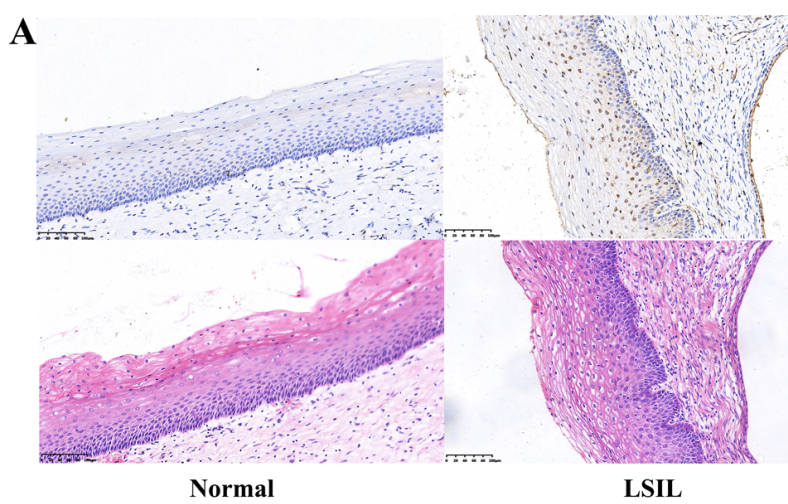

LSIL

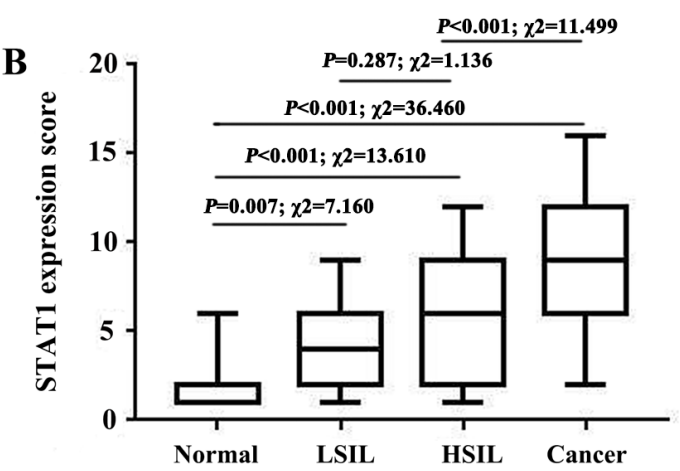

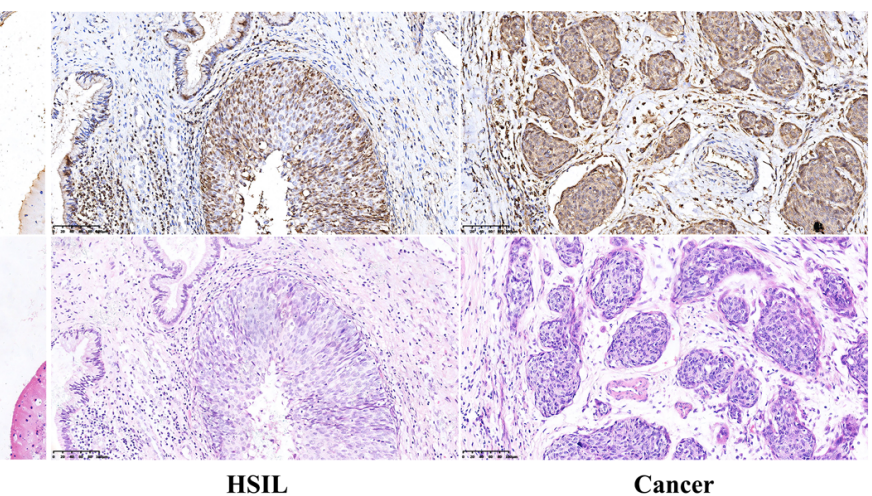

HSIL

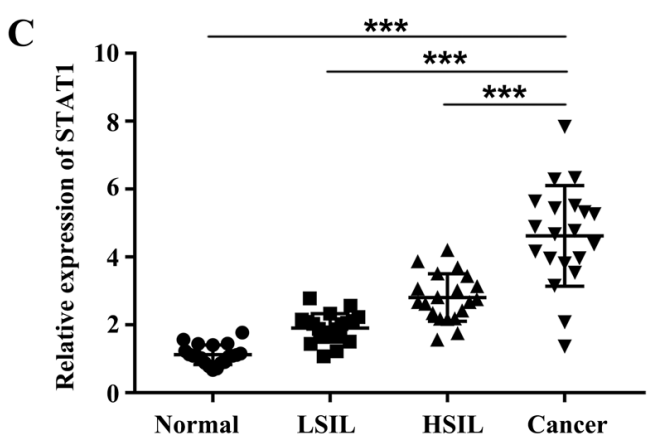

Figure 1. STAT1 expression in cervical tissues. (A) Immunohistochemical staining of STAT1 protein expression in normal cervical squamous epithelium, LSIL, HSIL and cervical squamous cell carcinoma. Magnification, x200. (B) Box plot of the immunohistochemical staining scores of STAT1 in normal tissues, LSIL, HSIL and cancer. Statistical analysis was performed using the $\chi^{2}$ test. (C) Total RNA was isolated to analyze STAT1 mRNA expression (20 cases per group). Data are presented as the mean $\pm \mathrm{SD} .{ }^{* * *} \mathrm{P} \leq 0.001$. HSIL, high-grade squamous intraepithelial lesion; LSIL, low-grade squamous intraepithelial lesion.

internal reference, fluorescent qPCR (SYBR-Green PCR kit; Qiagen $\mathrm{GmbH}$ ) was used to quantitate HPV16 DNA content in tissues. The amplification assay was performed on an I-cycler (Bio-Rad Laboratories, Inc.) with the following conditions: Pre-denaturation at $95^{\circ} \mathrm{C}$ for $2 \mathrm{~min}$, followed by 45 amplification cycles of $95^{\circ} \mathrm{C}$ for $5 \mathrm{sec}, 60^{\circ} \mathrm{C}$ for $1 \mathrm{~min}$, and with a final extension step at $72^{\circ} \mathrm{C}$ for $10 \mathrm{sec}$. The nucleotide sequences of the HPV16 L1 forward and reverse primer set used for amplification were 5'-ACTATTTTGGAGGACTGG-3' and 5'-TCTTTAGGTGCTGGAGGT-3', respectively. The relative amount of DNA was calculated using the $2^{-\Delta \Delta \mathrm{CT}}$ method.

Bioinformatics analysis. Gene Expression Profiling Interactive Analysis (GEPIA; http://gepia.cancer-pku.cn/) is a visualization website based on The Cancer Genome Atlas data (27). GEPIA was used to verify the differential expression of STAT1 in cervical squamous cell carcinoma (CESC) and normal tissues. Expression data were first transformed using the formula $\log 2(\mathrm{TPM}+1)$ for the differential analysis, and $\log 2 \mathrm{FC}$ was defined as the difference between the median of the tumor and the median of the normal tissue. An online KM plotter database (http://kmplot.com/analysis/, 2020.05.25) was used to analyze the overall survival rate of patients with different expression levels of STAT1 based on the clinical data of 304 patients with CESC (28). STAT1 was entered in the database to acquire CESC KM survival plots. The patients were divided into two cohorts according to the median expression of the gene. A logarithmic rank test was used for survival analysis. Hypothesis testing was performed, and the hazard ratio (HR) and $95 \% \mathrm{CI}$ were calculated.
Statistical analysis. Pearson's $\chi^{2}$ test was used to compare the characteristics of patients according to STAT1 expression and HPV viral load. All analyses were performed using the SPSS statistical package (version 25.0; IBM Corp.). Data were presented as the means \pm standard deviations. One-way ANOVA followed by Dunnett's post hoc test was used to determine statistically significant differences. To analyze the relationship between STAT1 protein expression and HPV viral load, Spearman's correlation analysis was performed for protein expression and HPV16 DNA load in the same samples using GraphPad Prism 6.0 software (GraphPad Software, Inc.). Receiver operating characteristic (ROC) curves and area under the curve (AUC) values were also calculated for STAT1 expression. All tests were two-tailed, and $\mathrm{P}<0.05$ was considered to indicate a statistically significant difference.

\section{Results}

Association between STAT1 expression and the extent of cervical lesions. To investigate the expression levels of STAT1, immunohistochemical staining of 141 cervical tissue samples was performed. The results revealed that STAT1 expression gradually increased with the progression of cervical lesions (Fig. 1A and B). STAT1 protein expression was observed in 10.71 (3/28), 41.18 (14/34), 53.06 (26/49) and 90.00\% (27/30) of normal cervical squamous epithelium, LSIL, HSIL and cervical squamous cell carcinoma samples, respectively (Table I). As shown in Fig. 1B, compared with normal tissues, STAT1 expression was significantly increased in LSIL $\left(\mathrm{P}=0.007 ; \chi^{2}=7.160\right)$, HSIL $\left(\mathrm{P}<0.001 ; \chi^{2}=13.610\right)$ and cancer 
Table I. Analysis of STAT1 expression and clinical factors of patients with cervical lesions.

\begin{tabular}{lccc}
\hline & & \multicolumn{2}{c}{ STAT1 } \\
\cline { 3 - 4 } Characteristics & Total, $\mathrm{n}$ & High expression, $\mathrm{n}$ & Low expression, $\mathrm{n}$ \\
\hline Age, years & & & 36 \\
$\leq 43$ & 63 & 27 & 35 \\
$>43$ & 78 & 43 & 25 \\
Lesion grade & & & 20 \\
Normal & 28 & 3 & 23 \\
LSIL & 34 & 14 & 3 \\
HSIL & 49 & 26 & 20.147 \\
Cancer & 30 & 27 & 3 \\
\hline
\end{tabular}

HSIL, high-grade squamous intraepithelial lesion; LSIL, low-grade squamous intraepithelial lesion.

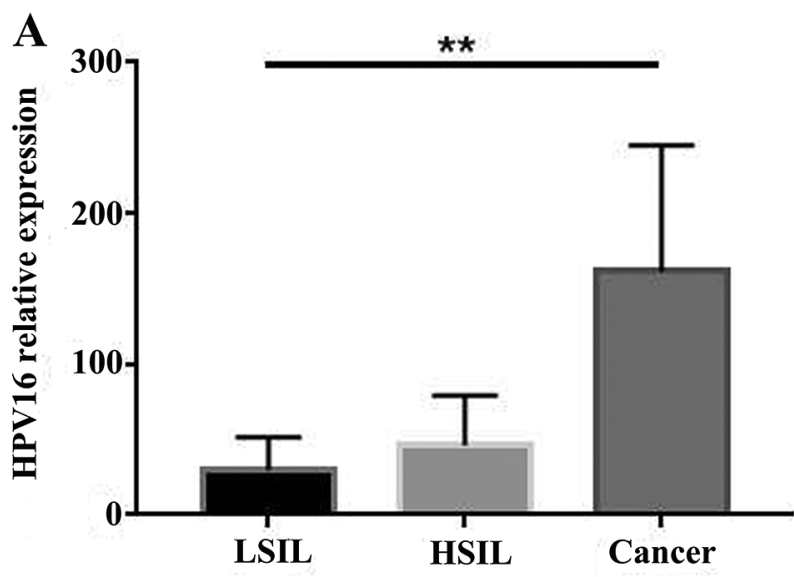

Figure 2. HPV16 viral load in cervical lesions. (A) HPV16 L1 reverse transcription-quantitative PCR using GAPDH as an internal control. HPV16 viral load was assessed using the $2^{-\Delta \Delta C T}$ method. One-way ANOVA followed by Dunnett's post hoc test was used for statistical analysis. (B) Correlation between HPV16 DNA load and STAT1 expression in different cervical lesions. Spearman's correlation analysis was performed. ${ }^{* *} \mathrm{P}<0.01$. HPV, human papillomavirus; HSIL, high-grade squamous intraepithelial lesion; LSIL, low-grade squamous intraepithelial lesion.

samples $\left(\mathrm{P}<0.001 ; \chi^{2}=36.460\right)$. The proportion of samples with positive STAT1 expression was higher in HSIL than in LSIL, although the difference was not statistically significant $\left(\mathrm{P}=0.287 ; \chi^{2}=1.136\right)$. Furthermore, the proportion of samples with positive STAT1 expression was higher in cervical cancer than in HSIL $\left(\mathrm{P}<0.001 ; \chi^{2}=11.499\right)$. Frozen tissue samples from 20 patients per group were selected for RT-qPCR to detect STAT1 mRNA expression. Compared with that in cancer samples, STAT1 mRNA expression was markedly decreased in normal, LSIL and HSIL samples (Fig. 1C). These findings demonstrated that STAT1 expression gradually increased with increasing severity of lesions in cervical lesion tissues.

Associations among HPV16 DNA load, extent of cervical lesions and STAT1 expression. To determine the distribution of HPV16 in the study samples, HPV16 L1 was detected. All normal group tissues were confirmed to be HPV16-negative. PCR detection was performed for 34 cases in the LSIL group, 49 in the HSIL group and 30 in the cancer group. The viral

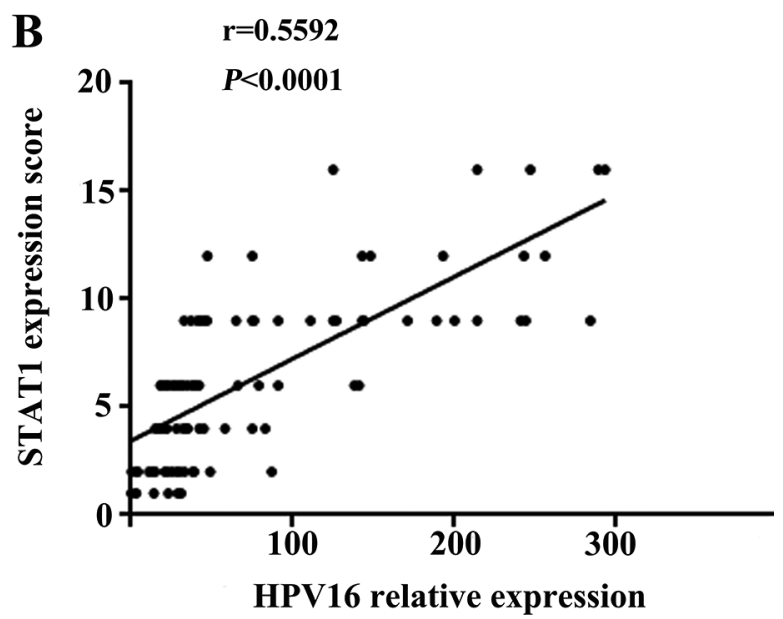

loads gradually increased from LSIL to HSIL to cervical squamous cell carcinoma, with mean values of $26.98 \pm 12.34$, $51.30 \pm 21.377$ and $169 \pm 79.47$, respectively. These data demonstrated that, as the disease progressed, HPV16 viral load gradually increased (Fig. 2A). Spearman's correlation analysis was used to analyze the correlation between HPV16 viral load and STAT1 expression. The results demonstrated that HPV16 viral load was positively correlated with STAT1 expression (Fig. 2B).

Associations among STAT1 expression, HPV16 viral load and the severity of cervical lesions in female patients. To elucidate the associations among STAT1 protein expression, HPV16 viral load (individually or in combination) and the severity of the cervical lesions in patients, ROC analysis was performed. The AUC values for distinguishing LSIL and HSIL samples from cervical cancer samples were 0.824 (95\% CI, 0.719-0.929) for STAT1 protein expression and 0.736 (95\% CI, 0.598-0.873) for HPV16 viral load (Table II). The AUC values for distinguishing LSIL samples from HSIL and cervical 
Table II. AUC values obtained from receiver operating characteristic curve analysis of individual or combined STAT1 expression and HPV16 viral load.

\begin{tabular}{|c|c|c|c|c|}
\hline Group & AUC (95\% CI) & Sensitivity, $\%$ & Specificity, \% & Threshold, \% \\
\hline \multicolumn{5}{|c|}{ LSIL and HSIL vs. cervical cancer } \\
\hline STAT1 & $0.824(0.719-0.929)$ & 72.0 & 85.2 & 37 \\
\hline HPV16 & $0.736(0.598-0.873)$ & 48.0 & 94.4 & 33 \\
\hline STAT1 and HPV16 & $0.849(0.749-0.949)$ & 76.0 & 85.2 & 29 \\
\hline \multicolumn{5}{|c|}{ LSIL vs. HSIL and cervical cancer } \\
\hline STAT1 & $0.711(0.592-0.830)$ & 43.4 & 88.5 & 76 \\
\hline HPV16 & $0.698(0.577-0.819)$ & 86.8 & 50.0 & 60 \\
\hline STAT1 and HPV16 & $0.739(0.627-0.852)$ & 56.6 & 84.6 & 69 \\
\hline \multicolumn{5}{|c|}{ HSIL vs. cervical cancer } \\
\hline STAT1 & $0.806(0.680-0.931)$ & 72.0 & 82.1 & 50 \\
\hline HPV16 & $0.713(0.563-0.863)$ & 48.0 & 96.4 & 51 \\
\hline STAT1 and HPV16 & $0.833(0.718-0.947)$ & 88.0 & 67.9 & 32 \\
\hline
\end{tabular}

AUC, area under the curve; HPV, human papillomavirus; HSIL, high-grade squamous intraepithelial lesion; LSIL, low-grade squamous intraepithelial lesion.

cancer samples were 0.711 (95\% CI, 0.592-0.830) for STAT1 protein expression and 0.698 (95\% CI, 0.577-0.819) for HPV16 viral load. The AUC values were improved by using a combination of the STAT1 protein expression and HPV16 viral load, with an effective distinction between LSIL and HSIL samples and cervical cancer samples (sensitivity, 76.0\%; specificity, $85.2 \%$ ) obtained using the mean change frequencies of STAT1 protein expression and HPV16 viral load (AUC, 0.849; 95\% CI, 0.749-0.949) when a $29 \%$ change frequency was used as the critical threshold (Fig. 3A; Table II). LSIL samples were distinguishable from cervical cancer and HSIL samples with a sensitivity of $56.6 \%$ and a specificity of $84.6 \%$ using the mean change frequencies of STAT1 protein expression and HPV16 viral load (AUC, 0.739; 95\% CI, 0.627-0.852) when a $69 \%$ change frequency was used as the critical threshold (Fig. 3B; Table II). Concordant trends were observed when distinguishing HSIL samples from cervical cancer samples using a $32 \%$ change frequency as the critical threshold, which yielded a sensitivity of $88.0 \%$ and a specificity of $67.9 \%$ (AUC, 0.833; 95\% CI, 0.718-0.947; Fig. 3C; Table II). The results revealed that STAT1 protein expression and HPV16 viral load, individually or in combination, could be used to differentiate between samples from patients with LSIL/HSIL and samples from patients with cervical cancer.

Bioinformatics analysis. The present study used the GEPIA database to analyze STAT1 expression in CESC tissues, and non-cancerous tissues, and identified that STAT1 expression was significantly higher in CESC tissues than in non-cancerous tissues (Fig. 4A). To clarify the effect of gene expression on survival time, Kaplan Meier-plotter, an online survival analysis tool, was used to analyze overall patient survival rates based on STAT1 expression using the clinical data of 304 patients with cervical cancer, of which there were 204 in the high expression group and 100 in the low expression group (HR, 0.54; $\mathrm{P}=0.015$; Fig. 4B). The results revealed that patients with cervical cancer with high STAT1 expression had longer survival.

\section{Discussion}

The present study revealed that increased STAT1 expression was associated with the progression of cervical lesions. STAT1 expression in cervical epithelial lesions was significantly higher than in normal cervical epithelium samples. In a study on breast cancer by Hix et al (29), STAT1 was also highly expressed in mouse breast cancer cells, enhancing the growth and invasiveness of cancer cells, while the growth and invasiveness were decreased in STAT1 knockout cancer cells. Therefore, high STAT1 expression could promote the initiation and progression of tumors. However, STAT1 is also widely recognized as a tumor suppressor, and some studies have found that its expression is increased in early cervical intraepithelial neoplasia (CIN) I/II cervical lesions, decreased in CIN III/cervical carcinoma in situ and significantly upregulated in invasive cervical cancer. These findings suggest that STAT1 may have dual effects on cervical tumorigenesis. In early stages, STAT1 has a protective effect, while it is carcinogenic in aggressive tumors $(30,31)$. STAT1 activation and enhanced expression in tumor cells are induced by different stimuli, such as IFN- $\alpha$, IFN- $\beta$, IFN- $\gamma$, the action of oncogenes or loss of tumor suppressors, interaction with the tumor stroma, or genotoxic agents (32). Phosphorylated STAT1 is the activated form of STAT1. The present study detected the total STAT1 protein levels because both phosphorylated and unphosphorylated proteins could affect the outcome of cervical cancer, as described previously for breast cancer (33) and ovarian cancer (34). Tumors with a high STAT1 levels express IFN-related DNA damage resistance signature, which is linked to therapy resistance. Translation, autophagy and apoptosis in tumor cells are also altered by STAT1 and may contribute to therapy resistance. 
LSIL and HSIL VS. cervical cancer

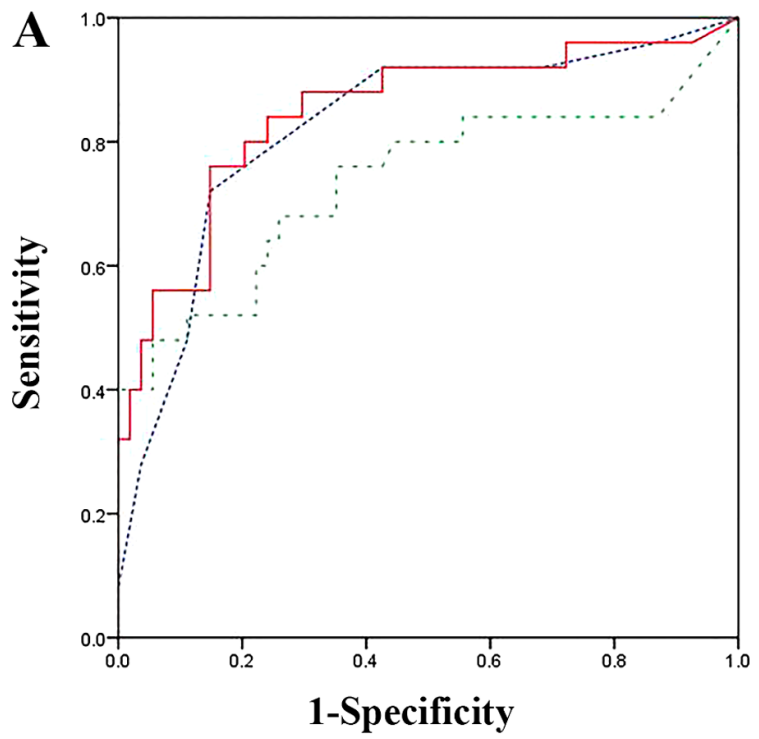

LSIL VS. HSIL and cervical cancer

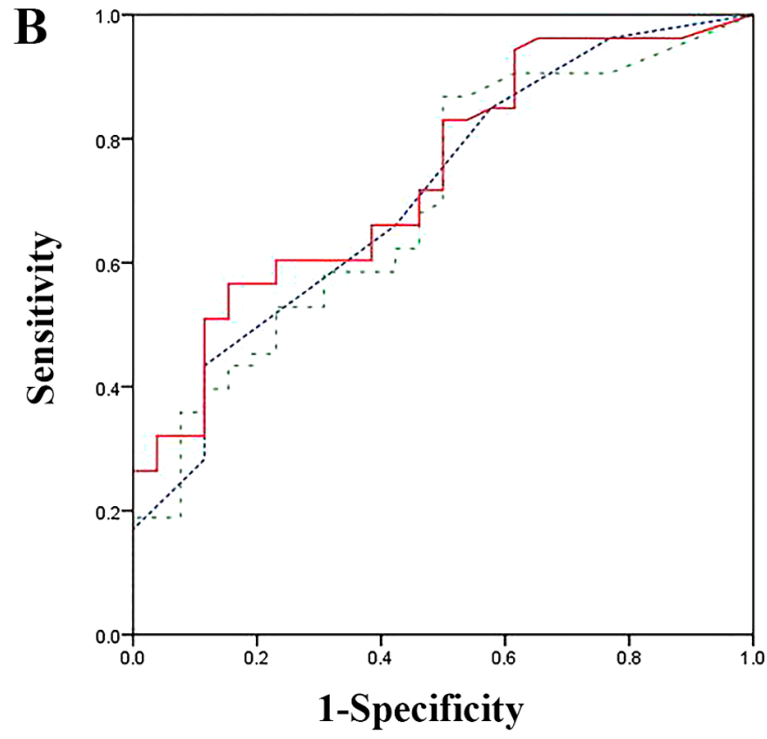

HSIL VS. cervical cancer

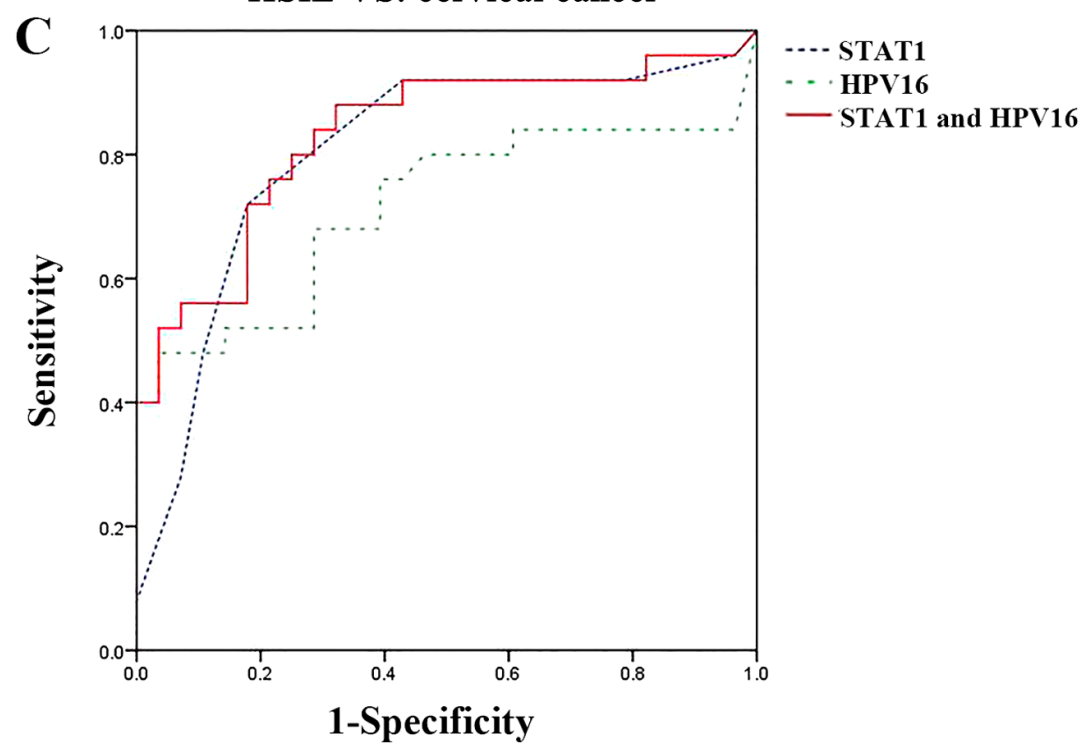

Figure 3. Receiver operating characteristic curves for the distinction between tissue samples using STAT1 expression and HPV16 viral load individually or in combination. (A) LSIL and HSIL vs. cervical cancer. (B) LSIL vs. HSIL and cervical cancer. (C) HSIL vs. cervical cancer. HPV, human papillomavirus; HSIL, high-grade squamous intraepithelial lesion; LSIL, low-grade squamous intraepithelial lesion.

Furthermore, STAT1 enhances the invasion and metastasis of tumor cells (32).

HPV is a circular double-stranded DNA virus and HR-HPV is closely associated with the occurrence of cervical cancer (35). As a product of the number of infected cells and the number of viruses per infected cell, HPV viral load is influenced by two main factors: The extent of an HPV infection on the cervical surface and the level of viral production in the infection area. Changes in viral load and the association of these changes with disease risk may indicate that there is a complex interaction between HPV and the human host, and thus such changes could potentially serve as an additional predictive marker for the outcome of infection (36). Among the HPV subtypes, the number of patients with cervical cancer caused by HPV16 is the greatest (37). In 1995, the World Health Organization defined HPV16 as a viral tumor promoter. Due to the carcinogenicity of HPV, detection technology for HPV has gradually improved, and accumulated evidence indicates that the sensitivity of HPV DNA detection (97.6\%) is higher than that of cytology. Therefore, in a number of countries, HPV DNA testing has been recommended as a primary screening method for cervical cancer (38). Furthermore, HPV16 DNA viral load in paraffin-embedded human cervical tissue samples was determined. Through qPCR, the present study revealed that viral load increased with the progression of cervical lesions. This is consistent with the findings of Camus et al (39) regarding cervical lesions. The development of cervical cancer is usually associated with an infection with HPV, particularly with a high-risk genotype of HPV (1). HPV16 is one of the most common genotypes, found in $>35 \%$ of patients with cervical cancer $(40,41)$. Changes in viral load and the associations of these changes with disease risk may imply a complex 
A

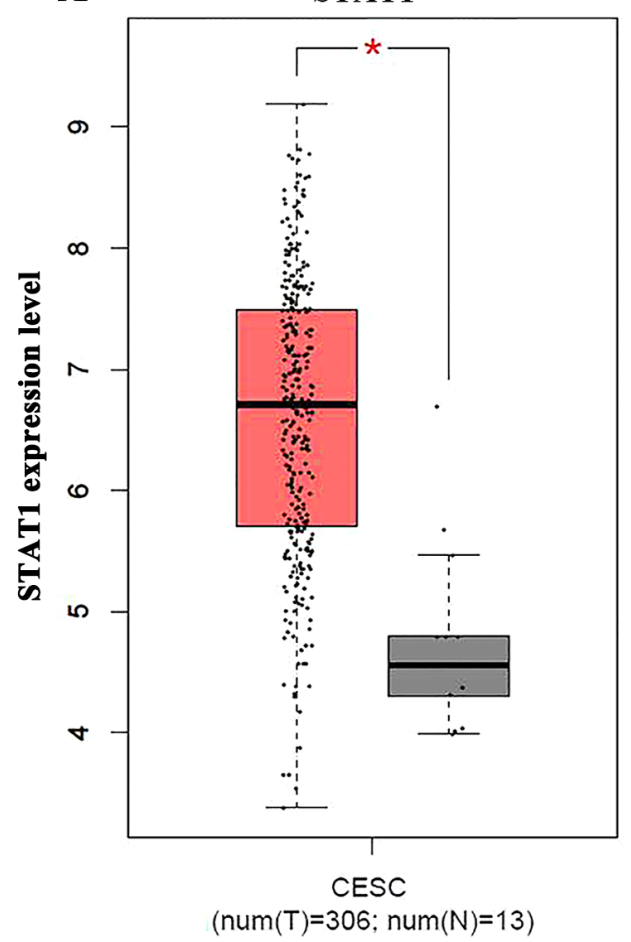

B

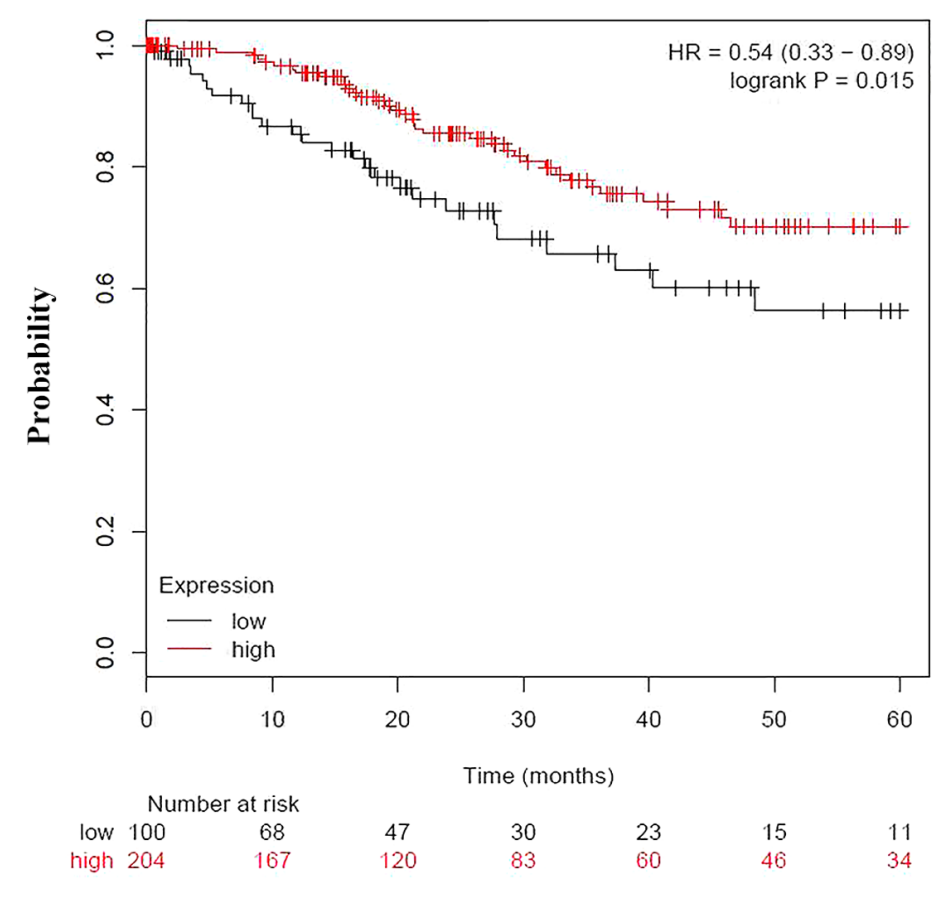

Figure 4. Bioinformatics analysis of STAT1 expression in cervical tissues. (A) Box plot of STAT1 expression in CESC tissues and non-cancerous tissues. (B) Survival curves of patients with cervical cancer grouped by STAT1 expression. ${ }^{*} \mathrm{P}<0.05$. CESC, cervical squamous cell carcinoma; HR, hazard ratio.

interaction between HPV and the human host, and potentially serve as an additional predictive marker for the outcomes of infection. A previous study suggested that HPV16 is not only particularly common, but also more oncogenic than other HPV types (42).

It has been reported that STAT1 expression is reduced in HFK cells transfected with the HPV16/31 genome and E6/E7 oncogenes (43). E6 and E7 serve as two key oncogenic transcription factors in the early stages of the HPV16 viral life cycle. E6 serves an important role in p53 degradation and can eliminate cell growth arrest, and E7 binds to and inactivates retinoblastoma protein (pRb), thus interfering with cell cycle regulation (44). E6 and E7 can synergistically promote the proliferation of infected cells, which promotes the occurrence and development of cervical cancer (35). By degrading p53, $\mathrm{pRb}$ and other tumor suppressor genes, these transformed keratinocytes have important effects on the expression of numerous target genes in cells (45).

The suppression of STAT1 by HPV proteins is necessary for the stable maintenance of viral episomes and genome amplification (11). By contrast, the present study revealed that STAT1 expression was positively correlated with HPV16 viral load in human cervical tissue samples. The reason for this relationship between STAT1 expression and HPV16 viral load is currently unclear, but three possible reasons were suggested. First, the cervical tissue consists of a complex microenvironment, including squamous epithelial cells, keratinocytes, macrophages, dendritic cells (DCs), neutrophils and natural killer (NK) cells. HPV16 infection is an ongoing process. Macrophages and DCs can recognize and phagocytize HPV16 viruses, induce an inflammatory response, and secrete pro-inflammatory cytokines, including
IFN- $\gamma$, interleukin- $1 \beta$ and TNF- $\alpha$. IFN- $\gamma$ can increase STAT1 expression, even in HPV-infected cells (11). Second, a previous study revealed that STAT3, a regulator of proliferation, apoptosis, inflammation and tumorigenesis, is positively associated with HPV16 and promotes cervical cancer progression (46). HPV16 E6 has also been demonstrated to increase and activate STAT3 (47). In addition, STAT3 has been reported to be able to cooperate with epidermal growth factor receptor and HER2 to regulate STAT1 transcriptional activation in breast cancer cells (48). Therefore, it was speculated that HPV16 mediates STAT1 transcription by activating STAT3 in cervical cancer. Third, in the case of HPV-positive oropharyngeal squamous cell carcinoma with non-destructive p53 mutations, the E6/E7 oncoprotein has been reported to be less likely to control intracellular target genes, including STAT1, by inhibiting p53 expression (49). It may be hypothesized that STAT1 expression may be associated with the type of p53 mutations inhibited by E6 in cervical cancer tissues.

The present bioinformatics analysis demonstrated that high STAT1 expression was associated with improved survival in patients with cervical cancer. However, the biological mechanisms behind this remain to be determined. Accumulating evidence has demonstrated that increased STAT1 expression promotes tumor progression in multiple types of cancer, including breast cancer (29), and STAT1 has been demonstrated to be associated with poor survival, as well as chemotherapy and radiotherapy resistance, in breast tumors (50). By contrast, previous studies have also demonstrated that STAT1, as a tumor suppressor (51), restrains angiogenesis and cell proliferation (52). Furthermore, increased STAT1 expression is associated with improved disease-free survival and 
overall survival in ovarian cancer (34), and could induce the expression of pro-survival genes and resistance to genotoxic stress in human squamous carcinoma cells (53). In addition, STAT1 serves important roles in determining the immune responses in the tumor microenvironment, and clinical findings have demonstrated the importance of immune responses in suppressing tumorigenesis (54). STAT1 can manipulate tumor immunoregulation by elevating the cytotoxicity of NK cells and $\mathrm{CD} 8^{+} \mathrm{T}$ cells and influencing the surface receptors on tumor cells, including programmed death-ligand 1 and cytotoxic T-lymphocyte associated protein 4 , which leads to the elimination of tumor cells by the immune system (32).

STAT1 can regulate various cellular processes, such as antimicrobial activities, tumor suppression, cell proliferation and cell death (32). Previous studies have demonstrated that STAT1 could mediate chemo- and radio-resistance during anticancer therapy $(55,56)$, while a recent cervical cancer study suggested that STAT1 may contribute to the enhanced radiosensitivity by regulating intracellular levels of reactive oxygen species (57). The present study revealed that the patients with high STAT1 expression had improved survival. STAT1 treatment may increase the radiosensitivity to improve the prognosis of patients with cervical cancer treated by radiotherapy, and improve the survival of patients with cervical cancer who had low STAT1 expression.

In summary, the present study demonstrated that STAT1 expression was positively associated with the progression of cervical lesions in HPV16-infected patients. The combined detection of STAT1 expression and HPV16 viral load may provide an improved method for distinguishing the grade of cervical lesions, and STAT1 expression was demonstrated to be associated with a favorable prognosis in cervical cancer. These findings will help to further understand the development of cervical lesions and may provide improved methods for tumor therapy.

\section{Acknowledgements}

Not applicable.

\section{Funding}

The present study was supported by the National Natural Science Foundation of China (grant no. 81171581), Natural Science Foundation of Liaoning Province (grant no. LFWK201710) and 345 Talent Project of Shengjing Hospital, China Medical University.

\section{Availability of data and materials}

The datasets used and/or analyzed during the present study are available from the corresponding author on reasonable request.

\section{Authors' contributions}

$\mathrm{SWu}, \mathrm{YW}$ and $\mathrm{ZS}$ were responsible for the conception and design of the study. SWu, YW and YZ collected the human samples. SWu, YW, YY, ML, YL, CC, SWa and MY performed experiments. SWu and $\mathrm{YW}$ analyzed results and interpreted data. ZS supervised the study. SWu and YL drafted the manuscript. YL, CC, MY, YZ and ZS revised the manuscript. All authors read and approved the final manuscript.

\section{Ethics approval and consent to participate}

The Ethics Committee of Shengjing Hospital, China Medical University approved the present study (approval no. 2019PS476K). Written informed consent was obtained from all patients.

\section{Patient consent for publication}

Not applicable.

\section{Competing interests}

The authors declare that they have no competing interests.

\section{References}

1. Cohen PA, Jhingran A, Oaknin A and Denny L: Cervical cancer. Lancet 393: 169-182, 2019.

2. Crosbie EJ, Einstein MH, Franceschi S and Kitchener HC: Human papillomavirus and cervical cancer. Lancet 382: 889-899, 2013.

3. Small W Jr, Bacon MA, Bajaj A, Chuang LT, Fisher BJ, Harkenrider MM, Jhingran A, Kitchener HC, Mileshkin LR, Viswanathan AN and Gaffney DK: Cervical cancer: A global health crisis. Cancer 123: 2404-2412, 2017.

4. Nitecki R and Feltmate CM: Human papillomavirus and nonhuman papillomavirus pathways to vulvar squamous cell carcinoma: A review. Curr Probl Cancer 42: 476-485, 2018.

5. Latsuzbaia A, Tapp J, Nguyen T, Fischer M, Arbyn M, Weyers S and Mossong J: Analytical performance evaluation of Anyplex II HPV28 and Euroarray HPV for genotyping of cervical samples. Diagn Microbiol Infect Dis 85: 318-322, 2016.

6. Tang A, Dadaglio G, Oberkampf M, Di Carlo S, Peduto L, Laubreton D, Desrues B, Sun CM, Montagutelli X and Leclerc C: $\mathrm{B}$ cells promote tumor progression in a mouse model of HPV-mediated cervical cancer. Int J Cancer 139: 1358-1371, 2016.

7. Chatzistamatiou K, Moysiadis T, Moschaki V, Panteleris N and Agorastos T: Comparison of cytology, HPV DNA testing and HPV 16/18 genotyping alone or combined targeting to the more balanced methodology for cervical cancer screening. Gynecol Oncol 142: 120-127, 2016.

8. Hong $\mathrm{S}$ and Laimins LA: The JAK-STAT transcriptional regulator, STAT-5, activates the ATM DNA damage pathway to induce HPV 31 genome amplification upon epithelial differentiation. PLoS Pathogens 9: e1003295, 2013.

9. Prabhavathy D, Vijayalakshmi R, Kanchana MP and Karunagaran D: HPV16 E2 enhances the expression of NF- $\kappa \mathrm{B}$ and STAT3 target genes and potentiates NF- $\kappa$ B activation by inflammatory mediators. Cell Immunol 292: 70-77, 2014.

10. Galloway DA, Morgan EL and Macdonald A: Autocrine STAT3 activation in HPV positive cervical cancer through a virus-driven Rac1-NFкB-IL-6 signalling axis. PLoS Pathogens 15: e1007835, 2019.

11. Hong S, Mehta KP and Laimins LA: Suppression of STAT-1 expression by human papillomaviruses is necessary for differentiation-dependent genome amplification and plasmid maintenance. J Virol 85: 9486-9494, 2011.

12. Manavi M, Hudelist G, Fink-Retter A, Gschwandtler-Kaulich D, Pischinger $\mathrm{K}$ and Czerwenka K: Gene profiling in Pap-cell smears of high-risk human papillomavirus-positive squamous cervical carcinoma. Gynecol Oncol 105: 418-426, 2007.

13. Verhoeven Y, Tilborghs S, Jacobs J, De Waele J, Quatannens D, Deben C, Prenen H, Pauwels P, Trinh XB, Wouters A, et al: The potential and controversy of targeting STAT family members in cancer. Semin Cancer Biol 60: 41-56, 2020.

14. Merlos-Suarez A, Barriga FM, Jung P, Iglesias M, Céspedes MV, Rossell D, Sevillano M, Hernando-Momblona X, da Silva-Diz V, Muñoz $\mathrm{P}$, et al: The intestinal stem cell signature identifies colorectal cancer stem cells and predicts disease relapse. Cell Stem Cell 8: 511-524, 2011. 
15. Chen J, Zhao J, Chen L, Dong N, Ying Z, Cai Z, Ji D, Zhang Y, Dong L, Li Y, et al: STAT1 modification improves therapeutic effects of interferons on lung cancer cells. J Transl Med 13: 293 , 2015.

16. Zhang Y, Molavi O, Su M and Lai R: The clinical and biological significance of STAT1 in esophageal squamous cell carcinoma. BMC Cancer 14: 791, 2014

17. Zhang X, Li X, Tan F, Yu N and Pei H: STAT1 Inhibits MiR-181a expression to suppress colorectal cancer cell proliferation through PTEN/Akt. J Cell Biochem 118: 3435-3443, 2017.

18. Chan SR, Vermi W, Luo J, Lucini L, Rickert C, Fowler AM, Lonardi S, Arthur C, Young LJ, Levy DE, et al: STAT1-deficient mice spontaneously develop estrogen receptor alpha-positive luminal mammary carcinomas. Breast Cancer Res 14: R16, 2012.

19. Arzt L, Kothmaier H, Halbwedl I, Quehenberger F and Popper HH: Signal transducer and activator of transcription 1 (STAT1) acts like an oncogene in malignant pleural mesothelioma. Virchows Arch 465: 79-88, 2014

20. Greenwood C, Metodieva G, Al-Janabi K, Lausen B, Alldridge L, Leng L, Bucala R, Fernandez N and Metodiev MV: Statl and CD74 overexpression is co-dependent and linked to increased invasion and lymph node metastasis in triple-negative breast cancer. J Proteomics 75: 3031-3040, 2012.

21. Zhu H, Wang Z, Xu Q, Zhang Y, Zhai Y, Bai J, Liu M, Hui Z and $\mathrm{Xu}$ N: Inhibition of STAT1 sensitizes renal cell carcinoma cells to radiotherapy and chemotherapy. Cancer Biol Ther 13: 401-407, 2012

22. Duarte CW, Willey CD, Zhi D, Cui X, Harris JJ, Vaughan LK, Mehta T, McCubrey RO, Khodarev NN, Weichselbaum RR and Gillespie GY: Expression signature of IFN/STAT1 signaling genes predicts poor survival outcome in glioblastoma multiforme in a subtype-specific manner. PLoS One 7: e29653, 2012.

23. Magkou C, Giannopoulou I, Theohari I, Fytou A, Rafailidis P, Nomikos A, Papadimitriou C and Nakopoulou L: Prognostic significance of phosphorylated STAT-1 expression in premenopausal and postmenopausal patients with invasive breast cancer. Histopathology 60: 1125-1132, 2012

24. Liu SS, Leung RCY, Chan KKL, Cheung ANY and Ngan HYS Evaluation of a newly developed GenoArray human papillomavirus (HPV) genotyping assay and comparison with the Roche Linea Array HPV genotyping assay. J Clin Microbiol 48: 758-764, 2010.

25. Livak KJ and Schmittgen TD: Analysis of relative gene expression data using real-time quantitative PCR and the 2(-Delta Delta C(T)) method. Methods 25: 402-408, 2001.

26. Biesaga B, Janecka A, Mucha-Malecka A, Adamczyk A Szostek S, Słonina D, Halaszka K and Przewoźnik M: HPV16 detection by qPCR method in relation to quantity and quality of DNA extracted from archival formalin fixed and paraffin embedded head and neck cancer tissues by three commercially available kits. J Virol Methods 236: 157-163, 2016.

27. Tang Z, Li C, Kang B, Gao G, Li C and Zhang Z: GEPIA: A web server for cancer and normal gene expression profiling and interactive analyses. Nucleic Acids Res 45: W98-W102, 2017.

28. Zhang J, Zheng Z, Zheng J, Xie T, Tian Y, Li R, Wang B, Lin J, $\mathrm{Xu}$ A, Huang X and Yuan Y: Epigenetic-Mediated downregulation of zinc finger protein 671 (ZNF671) predicts poor prognosis in multiple solid tumors. Front Oncol 9: 342, 2019

29. Hix LM, Karavitis J, Khan MW, Shi YH, Khazaie K and Zhang M: Tumor STAT1 transcription factor activity enhances breast tumor growth and immune suppression mediated by myeloid-derived suppressor cells. J Biol Chem 288: 11676-11688, 2013.

30. Rajkumar T, Sabitha K, Vijayalakshmi N, Shirley S, Bose MV, Gopal $\mathrm{G}$ and Selvaluxmy G: Identification and validation of genes involved in cervical tumourigenesis. BMC Cancer 11: 80, 2011

31. Yi Y, Fang Y, Wu K, Liu Y and Zhang W: Comprehensive gene and pathway analysis of cervical cancer progression. Oncol Lett 19: 3316-3332, 2020

32. Meissl K, Macho-Maschler S, Muller M and Strobl B: The good and the bad faces of STAT1 in solid tumours. Cytokine 89: 12-20, 2017.

33. Gujam FJ, McMillan DC and Edwards J: The relationship between total and phosphorylated STAT1 and STAT3 tumour cell expression, components of tumour microenvironment and survival in patients with invasive ductal breast cancer. Oncotarget 7: 77607-77621, 2016.

34. Josahkian JA, Saggioro FP, Vidotto T, Ventura HT, Candido Dos Reis FJ, de Sousa CB, Tiezzi DG, de Andrade JM, Koti $\mathrm{M}$ and Squire JA: Increased STAT1 expression in high grade serous ovarian cancer is associated with a better outcome. Int J Gynecol Cancer 28: 459-465, 2018.
35. Guo Y, Meng X, Ma J, Zheng Y, Wang Q, Wang Y and Shang $\mathrm{H}$ Human papillomavirus 16 E6 contributes HIF-1 $\alpha$ induced Warburg effect by attenuating the VHL-HIF-1 $\alpha$ interaction. Int J Mol Sci 15: 7974-7986, 2014

36. Sun Z, Zhang R, Liu Z, Liu C, Li X, Zhou W, Yang L, Ruan Q and Zhang X: Development of a fluorescence-based multiplex genotyping method for simultaneous determination of human papillomavirus infections and viral loads. BMC Cancer 15: 860, 2015.

37. Graham SV: The human papillomavirus replication cycle, and its links to cancer progression: A comprehensive review. Clin Sci (Lond) 131: 2201-2221, 2017.

38. Franceschi S, Denny L, Irwin KL, Jeronimo J, Lopalco PL, Monsonego J, Peto J, Ronco G, Sasieni P and Wheeler CM: Eurogin 2010 roadmap on cervical cancer prevention. Int J Cancer 128: 2765-2774, 2011.

39. Camus C, Vitale S, Loubatier C, Pénaranda G, Khiri H, Plauzolles A, Carcopino X, Halfon P and Giordanengo V: Quantification of HPV16 E6/E7 mRNA spliced isoforms viral load as a novel diagnostic tool for improving cervical cancer screening. J Clin Med 7: 530, 2018

40. Li N, Franceschi S, Howell-Jones R, Snijders PJ and Clifford GM: Human papillomavirus type distribution in 30,848 invasive cervical cancers worldwide: Variation by geographical region, histological type and year of publication. Int J Cancer 128: 927-935, 2011.

41. de Sanjose S, Quint WG, Alemany L, Geraets DT, Klaustermeier JE, Lloveras B, Tous S, Felix A, Bravo LE, Shin HR, et al: Human papillomavirus genotype attribution in invasive cervical cancer: A retrospective cross-sectional worldwide study. Lancet Oncol 11: 1048-1056, 2010

42. Kjaer SK, Frederiksen K, Munk C and Iftner T: Long-term absolute risk of cervical intraepithelial neoplasia grade 3 or worse following human papillomavirus infection: Role of persistence. J Natl Cancer Inst 102: 1478-1488, 2010.

43. Hong S, Mehta KP and Laimins LA: Suppression of STAT-1 expression by human papillomaviruses is necessary for differentiation-dependent genome amplification and plasmid maintenance. J Virol 85: 9486-9494, 2011

44. Bordignon V, Di Domenico EG, Trento E, D'Agosto G, Cavallo I, Pontone M, Pimpinelli F, Mariani L and Ensoli F: How human papillomavirus replication and immune evasion strategies take advantage of the host dna damage repair machinery. Viruses 9: 390,2017

45. Smeets SJ, van der Plas M, Schaaij-Visser TB, van Veen EA, van Meerloo J, Braakhuis BJ, Steenbergen RD and Brakenhoff RH: Immortalization of oral keratinocytes by functional inactivation of the $\mathrm{p} 53$ and $\mathrm{pRb}$ pathways. Int J Cancer 128: 1596-1605, 2011.

46. Shukla S, Shishodia G, Mahata S, Hedau S, Pandey A, Bhambhani S, Batra S, Basir SF, Das BC and Bharti AC: Aberrant expression and constitutive activation of STAT3 in cervical carcinogenesis: Implications in high-risk human papillomavirus infection. Mol Cancer 9: 282, 2010.

47. Shukla S, Mahata S, Shishodia G, Pandey A, Tyagi A, Vishnoi K, Basir SF, Das BC and Bharti AC: Functional regulatory role of STAT3 in HPV16-mediated cervical carcinogenesis. PLoS One 8: e67849, 2013.

48. Han W, Carpenter RL, Cao X and Lo HW: STAT1 gene expression is enhanced by nuclear EGFR and HER2 via cooperation with STAT3. Mol Carcinog 52: 959-969, 2013.

49. Oh JE, Kim JO, Shin JY, Zhang XH, Won HS, Chun SH, Jung CK, Park WS, Nam SW, Eun JW and Kang JH: Molecular genetic characterization of p53 mutated oropharyngeal squamous cell carcinoma cells transformed with human papillomavirus E6 and E7 oncogenes. Int J Oncol 43: 383-393, 2013.

50. Khodarev N, Ahmad R, Rajabi H, Pitroda S, Kufe T, McClary C, Joshi MD, MacDermed D, Weichselbaum R and Kufe D: Cooperativity of the MUC1 oncoprotein and STAT1 pathway in poor prognosis human breast cancer. Oncogene 29: 920-929, 2010.

51. Lynch RA, Etchin J, Battle TE and Frank DA: A small-molecule enhancer of signal transducer and activator of transcription 1 transcriptional activity accentuates the antiproliferative effects of IFN-gamma in human cancer cells. Cancer Res 67: 1254-1261, 2007.

52. Klampfer L: Signal transducers and activators of transcription (STATs): Novel targets of chemopreventive and chemotherapeutic drugs. Curr Cancer Drug Targets 6: 107-121, 2006.

53. Khodarev NN, Beckett M, Labay E, Darga T, Roizman B and Weichselbaum RR: STAT1 is overexpressed in tumors selected for radioresistance and confers protection from radiation in transduced sensitive cells. Proc Proc Natl Acad Sci USA 101: 1714-1719, 2004 
54. Koebel CM, Vermi W, Swann JB, Zerafa N, Rodig SJ, Old LJ, Smyth MJ and Schreiber RD: Adaptive immunity maintains occult cancer in an equilibrium state. Nature 450: 903-907, 2007.

55. Khodarev NN, Roizman B and Weichselbaum RR: Molecular pathways: Interferon/stat1 pathway: Role in the tumor resistance to genotoxic stress and aggressive growth. Clin Cancer Res 18: 3015-3021, 2012

56. Hui Z, Tretiakova M,Zhang Z,Li Y, Wang X, Zhu JX, Gao Y, Mai W, Furge K, Qian CN, et al: Radiosensitization by inhibiting STAT1 in renal cell carcinoma. Int J Radiat Oncol Biol Phys 73: 288-295, 2009.
57. Buttarelli M, Babini G, Raspaglio G, Filippetti F, Battaglia A Ciucci A, Ferrandina G, Petrillo M, Marino C, Mancuso M, et al: A combined ANXA2-NDRG1-STAT1 gene signature predicts response to chemoradiotherapy in cervical cancer. J Exp Clin Cancer Res 38: 279, 2019.

(i) This work is licensed under a Creative Commons C. AO NO Attribution-NonCommercial-NoDerivatives 4.0 International (CC BY-NC-ND 4.0) License. 